\title{
Development of Carbon Nanotube Based Reflection Type X-ray Source
}

\author{
H.Y. CHOI ${ }^{a}$, J.U. KIM ${ }^{a, *}$ AND C.J. LEE ${ }^{b}$ \\ ${ }^{a}$ Centre for Pioneering Medical-Physics Research (CPMR), Korea Electrotechnology \\ Research Institute, 1271-19 Sa-dong, Ansan 426-901, Korea \\ ${ }^{b}$ College of Engineering, Korea University, Anam-dong, 136-701 Seoul, Korea
}

\begin{abstract}
X-ray imaging technology is a useful and leading medical diagnostic tool for health care professionals to diagnose disease in human body. Carbon nanotube based X-ray source, which we have developed in this study, could be also useful and supply integrated diagnostic X-ray imaging tool in diagnosis. Conventionally, thermionic type of tungsten filament X-ray tube is widely employed in the field of biomedical and industrial application fields. However, intrinsic problems, such as poor emission efficiency, low imaging resolution, and high electrical energy consumption etc., may cause the limitation of using the X-ray tube. To fulfill the current market requirement, specifically for medical diagnostic field, we have developed rather a portable and compact carbon nanotube based $\mathrm{X}$-ray device in which microfocus high imaging resolution can be feasible.
\end{abstract}

PACS numbers: 75.47.Lx, 75.47.Gk, 73.50.Fq

\section{Introduction}

It is known that today's diagnostic service providers are forced to provide rather a portable X-ray system, which supports the highest radiographic quality with less economical expense. Therefore, in the current study, we report on the development of a new X-ray source by using nanostructured carbon nanotube (CNT) based cold electron emitter. Such an X-ray source provides many advantages over the conventional electron source for many applications [1-5]. Since the CNT based portable X-ray system that we have developed contains a direct digital imaging sensor or detector, real time imaging is feasible and it, therefore, addresses a broad range of clinical and technological applications in general purpose of radiography.

\section{CNT emitter synthesis}

In order to synthesize double wall carbon nanotubes (DWCNTs) using $\mathrm{CH}_{4}$, a catalyst was prepared by embedding the $\mathrm{Fe}-\mathrm{Mo}$ bimetallic catalyst onto $\mathrm{MgO}$ powder. $\mathrm{Fe}-\mathrm{Mo} / \mathrm{MgO}$ catalyst was prepared according to the following procedure. A mixture of $\mathrm{Fe}\left(\mathrm{NO}_{3}\right)_{3} \cdot 9 \mathrm{H}_{2} \mathrm{O}(99 \%$, Aldrich) and Mo solution (Aldrich, ICP/DCP standard solution, $10 \mathrm{mg} / \mathrm{ml}$ of $\mathrm{Mo}$ in $\mathrm{H}_{2} \mathrm{O}$ ) was dissolved in DI water for $1 \mathrm{~h}$. The mixed Fe-Mo solution was introduced to the solution of $\mathrm{MgO}$ powder and DI water followed by sonication for $1 \mathrm{~h}$. The molar ratio of the catalyst was $\mathrm{Fe}: \mathrm{Mo}: \mathrm{MgO}=1: 0.1: 12$. After drying, the material

* corresponding author; e-mail: jukim@keri.re.kr was baked at $150^{\circ} \mathrm{C}$ for $15 \mathrm{~h}$ in vacuum and then ground in a mortar, and finally calcined in a quartz furnace at $700^{\circ} \mathrm{C}$ for $2 \mathrm{~h}$ in $\mathrm{O}_{2}$ ambient. The DWCNTs were synthesized in a quartz tube reactor (inner diameter: $70 \mathrm{~mm}$, length: $700 \mathrm{~mm}$ ). About $200 \mathrm{mg}$ of the supported Fe-Mo catalyst was placed in a quartz boat at the center of the reactor tube. The quartz tube was heated up to $900^{\circ} \mathrm{C}$ in $\mathrm{Ar}$ atmosphere. Subsequently, $\mathrm{CH}_{4}(300 \mathrm{sccm})$ and a mixture of $\mathrm{Ar}(500 \mathrm{sccm})$ and $\mathrm{H}_{2}(100 \mathrm{sccm})$ were introduced into the reactor. After $20 \mathrm{~min}$, the reactor was cooled to room temperature in Ar atmosphere. The as-synthesized DWCNTs were purified using a two-step purification process. Firstly, as-synthesized DWCNTs were oxidized in a furnace at $350^{\circ} \mathrm{C}$ in air atmosphere for 30 min to remove the amorphous carbon material on the surface of DWCNTs. Secondly, the oxidized DWCNTs were soaked in a diluted acetic acid solution at room temperature to remove catalyst particles existing in carbon materials. The DWCNT suspension was collected by membrane filtration (pore size: $0.2 \mu \mathrm{m}$ ) and washed with distilled water several times. Finally, we obtained the purified DWCNTs (non-annealed DWCNTs). After collecting the purified DWCNTs, the high temperature thermal annealing was carried out at $1300^{\circ} \mathrm{C}$ for $1 \mathrm{~h}$ in vacuum condition of $1.0 \times 10^{-5}$ Torr in order to reduce the defects in DWCNTs, resulting in improvement of crystallinity of DWCNTs.

\section{Results and discussion}

To evaluate the field emission properties of DWCNTs, the planar typed CNT field emitter was fabricated on the circular stainless steel substrate (SUS304) with $5 \mathrm{~mm}$ diameter. The Ag paste was coated on the stainless steel 
substrate and then the DWCNTs were spread on the Ag paste/stainless steel substrate using a sieving method. The DWCNTs deposited on the substrates were vertically aligned by a mechanical method containing attaching and peeling off a tape on the surface of DWCNTs. Field emission properties were measured in a vacuum chamber at pressure of less than $2 \times 10^{-7}$ Torr. The anode was a stainless steel plate with a dimension of $2 \times 2 \mathrm{~cm}^{2}$ but for the emission pattern, ITO substrate was used to the anode electrode. The gap between the cathode and the anode was $400 \mu \mathrm{m}$. To obtain stable field emission properties, an electrical annealing process was performed at the emission current of $1.0 \mathrm{~mA} / \mathrm{cm}^{2} \mathrm{sev}-$ eral times. After removing some protruding DWCNTs from the DWCNT emitter using the electrical annealing, the field emission properties were evaluated. In order to reduce edge emission from the circular disk-typed emitter, we ground the edge of the circular disk-type metal substrate and maintained the edge angle of the circular disk-typed emitter about $45^{\circ}$ before coating the $\mathrm{Ag}$ paste. After grinding the edge of the circular disk-typed emitter, we could obtain uniform emission from the emitter. The turn-on field of DWCNTs is about $2.4 \mathrm{~V} / \mathrm{m}$ at the emission current density of $0.1 \mu \mathrm{A} / \mathrm{cm}^{2}$ and the threshold field is about $3.15 \mathrm{~V} / \mu \mathrm{m}$ at the emission current density of $1.0 \mathrm{~mA} / \mathrm{cm}^{2}$. Especially, the DWCNT emitter shows much higher emission current density of $87.8 \mathrm{~mA} / \mathrm{cm}^{2}$ at the applied electric field about $5.5 \mathrm{~V} / \mathrm{m}$, which indicates a high enough current level (i.e., $17.2 \mathrm{~mA}$ for $5 \mathrm{~mm} \mathrm{CNT}$ emitter) for X-ray source application.

In the simulation, a typical triode model of a CNT emitter based X-ray source was employed. To fabricate rather a flawless CNT emitter X-ray source it is essential to perform computer simulation in advance. Basically, the triode model of the X-ray source consists of a CNT emitter as a cathode, a metal grid mesh as a gate, which functions to extract electrons from the tip of the CNT emitter and to modulate the electron beam somewhat, and finally an anode. But, in this model, electrostatic lenses are included to focus the electron beam with a microlevel onto the anode target. The important CNT $\mathrm{X}$-ray source parameters, such as the diameter of the CNT emitter and the distance between the anode target to the CNT emitter source were $5 \mathrm{~mm}$ and $23 \mathrm{~mm}$, respectively. Although it is not shown here, the typical $\mathrm{kVp}$ of the fabricated CNT X-ray source was measured with $40 \mathrm{kVp}$.

Figure 1 shows the typical simulation results of the time of flight (TOF) of the electron beam trajectory exiting from the CNT emitter. The diameter of the CNT emitter and the distance between the CNT emitter to the anode target was fixed to $5 \mathrm{~mm}$ and $23 \mathrm{~mm}$, respectively. As shown in Fig. 1, the focal point of the electron beam (i.e., specific distance at which the smallest spot size of the electron beam found) tends to increase with increasing the focusing lens voltage. In addition, at higher lens voltage, some fraction of the electron beam did not focus but loose instead as seen in Fig. 1b. In the simulation,

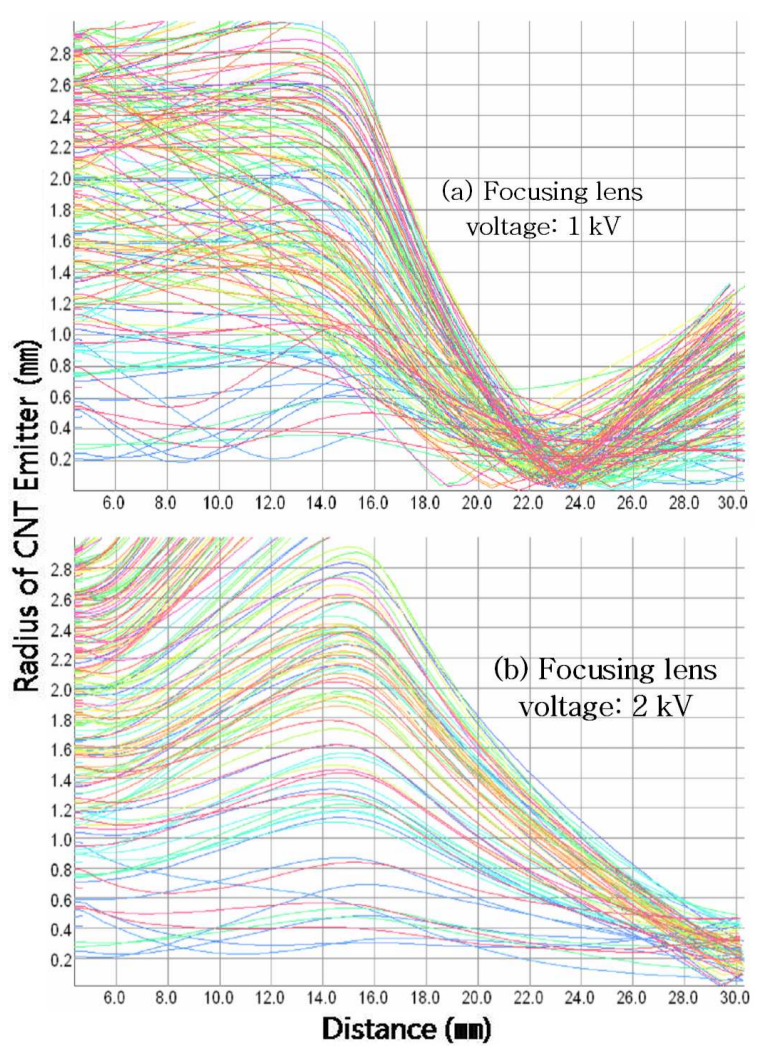

Fig. 1. Examples of typical simulation results of the time of flight (TOF) trajectory of electron beam using the triode model. In the simulation, the diameter of the CNT emitter was $5 \mathrm{~mm}$ and the distance between the anode target to the CNT emitter source was $23 \mathrm{~mm}$.

the calculated electron beam spot size is approximately less than $700 \mu \mathrm{m}$ at the anode target.

The triode model simulated in this study is applied to design and fabricate a prototype of a CNT-emitter based X-ray source. Although it is not shown, here it was housed in the vacuum chamber, which is sustained with low pressure of $10^{-7}$ Torr. And also, it is automatically operated by computer (i.e., Lab View program) to supply and maintain voltages and currents and also to obtain $\mathrm{X}$-ray images.

\section{Conclusions}

Using the CNT X-ray source, X-ray images of a finger bone and teeth in human body were obtained. The trabeculation shape in finger bone is clearly observed in Fig. 2a. To obtain the finger bone image, tube currents of $250 \mu \mathrm{A}$ at $42 \mathrm{kV}$ tube voltage was applied. The human tooth image (Fig. 2b), however, is somewhat unclear because the supplied voltage was limited to maximally $50 \mathrm{kV}$ in the system. It should be noted that normally $60-70 \mathrm{kV}$ of tube voltage is required in dental imaging. Considering this, if the tube voltage is over $60 \mathrm{kV}$ then clearer image can be feasible. Based on these preliminary results, it can be concluded that the CNT based 
(a)

(b)
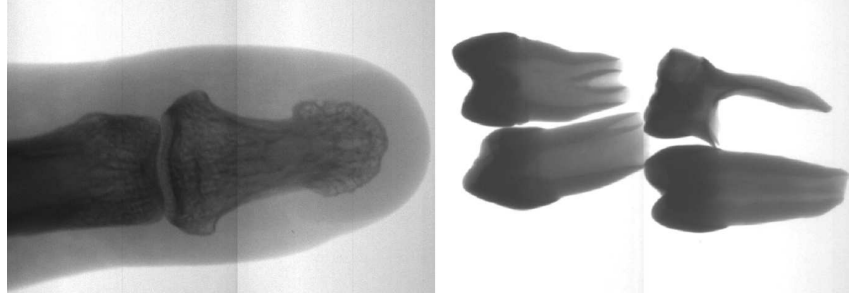

Fig. 2. Imaging of human finger (a) and teeth (b) using the fabricated CNT X-ray source.

X-ray source may be applicable to dental diagnosis and orthopedics in near future.

\section{Acknowledgments}

This work was partially supported by grants from Seoul R\&BD program with contract number CR070054.

\section{References}

[1] W. Zhu, C. Bower, O. Zhou, G. Kochanski, S. Jin, Appl. Phys. Lett. 75, 873 (1999).

[2] J. Zhang, Y. Cheng, Y.Z. Lee, B. Gao, Q. Qiu, W.L. Lin, D. Lalush, J.P. Lu, O. Zhou, Rev. Sci. Instrum. 76, 094301 (2005).

[3] G.Z. Yue, Q. Qiu, Bo. Ga, Y. Chaeng, J. Zhang, H. Shimoda, S. Chang, J.P. Lu, O. Zhou, Appl. Phys. Lett. 81, 355 (2002).

[4] F. Nicolaescu, J. Okuyama, J. Vac. Sci. Technol. A 1, 2369 (1997).

[5] H. Sugie, M. Tanemura, V. Filip, K. Takahashi, F. Okuyama, Appl. Phys. Lett. 78, 2578 (2001). 